\title{
BMJ Open Association between carotid intima- media thickness and bone mineral density: a cross-sectional study in Vietnamese men and women aged 50 years and older
}

\author{
Lich T Nguyen, ${ }^{1,2}$ Vinh N Pham, ${ }^{3}$ Phuong M N Chau, ${ }^{1}$ Lan T Ho-Pham,,${ }^{1,4}$ \\ Tuan V Nguyen ${ }^{5,6}$
}

To cite: Nguyen LT, Pham VN, Chau PMN, et al. Association between carotid intimamedia thickness and bone mineral density: a crosssectional study in Vietnamese men and women aged 50 years and older. BMJ Open 2019;9:e028603. doi:10.1136/ bmjopen-2018-028603

- Prepublication history and additional material for this paper are available online. To view these files, please visit the journal online (http://dx.doi org/10.1136/bmjopen-2018028603).

Received 17 December 2018 Revised 17 July 2019 Accepted 22 August 2019

Check for updates

(c) Author(s) (or their employer(s)) 2019. Re-use permitted under CC BY-NC. No commercial re-use. See rights and permissions. Published by BMJ.

For numbered affiliations see end of article.

Correspondence to

Dr Lan T Ho-Pham;

hophamthuclan@tdt.edu.vn

\section{ABSTRACT}

Objectives The association between osteoporosis and atherosclerosis remains controversial. We sought to define the relationship between carotid intima-media thickness and bone mineral density (BMD) in individuals of Vietnamese background.

Design and setting Cross-sectional study in Ho Chi Minh City, Vietnam.

Participants The study involved 1460 individuals (559 men) aged 50 years and older (average age 59 years) who were randomly recruited from the community.

Outcome measures BMD at the femoral neck and lumbar spine was measured by dual-energy X-ray absorptiometry (Hologic, Waltham, Massachusetts, USA). Carotid intima-media thickness (cIMT) was measured using a Philips Ultrasonography (HD7XE). The presence of atherosclerotic plaque was ascertained for each individual. The association between cIMT and BMD was analysed by a multiple linear regression model.

Results In unadjusted analysis, cIMT was positively associated with femoral neck BMD in men $(p=0.005)$, but not in women ( $p=0.242)$. After adjusting for age, smoking, diabetes and hypertension, the association remained statistically significant in men (partial $R^{2}=0.005 ; p=0.015$ ) but not in women (partial $R^{2}=0.008 ; p=0.369$ ). When the analysis was limited to individuals aged 60 years and older, the association between cIMT and BMD was no longer statistically significant. There was no statistically significant association between cIMT and lumbar spine BMD in either men or women.

Conclusions In Vietnamese individuals aged 50 years and older, there is a clinically non-significant but statistically significant association between carotid intima-media thickness and BMD in men, not in women.

\section{INTRODUCTION}

Several lines of evidence suggest that osteoporosis and atherosclerosis are linked. At the population level, the risks of osteoporosis and atherosclerosis increase with advancing age. The two conditions are also associated with reduced oestrogen (in women). Individuals
Strengths and limitations of this study

- This study was based on a large and well-characterised cohort which was randomly recruited from the general population.

- The measurements of bone mineral density and atherosclerosis were performed with state-of-the-art equipment, with high accuracy and reliability.

- The study did not consider other measures of atherosclerosis such as pulse wave velocity, aortic calcification index and endothelial function which have previously been found to be associated with bone mineral density.

- The cross-sectional nature of the study does not allow a causal inference to be made for the association between carotid atherosclerosis and osteoporosis.

with low bone mineral density (BMD) have increased risk of mortality due to stroke. ${ }^{1}$ In human, osteoprotegerin-a key regulator of bone metabolism-is associated with vascular calcification $^{2}$ and coronary artery disease. ${ }^{3}$ Increased serum levels of osteoprotegerin were also associated with an increased prevalence of aortic plaque and coronary artery calcification. ${ }^{4}$ Moreover, bone turnover markers such as carboxy-terminal telopeptide of type I collagen are also positively associated with carotid artherosclerosis. ${ }^{5}$ Thus, the biologic evidence for the linkage between osteoporosis and atherosclerosis is strong.

Nevertheless, epidemiological studies have yielded conflicting results of the association between osteoporosis and atherosclerosis. The association between the two diseases can be assessed in terms of surrogate measures such as BMD (for osteoporosis), carotid intima-media thickness (cIMT), pulse wave velocity, ankle-arm index, aortic calcification index and endothelial function. While most 
studies in Caucasian populations found an inverse association between cIMT and BMD, ${ }^{6-8}$ other studies in Asian populations found no statistically significant correlation between cIMT and BMD. ${ }^{9}{ }^{10}$ Still, a study in premenopausal women found a positive association between cIMT and hip BMD. ${ }^{11}$ The discrepancy in findings could be due to study design, characteristics of participants and methods of measurement. Apart from the UK-based study, ${ }^{11}$ most previous studies were based on relatively small sample sizes which limit the power to identify a fine association. Moreover, the association between cIMT and BMD could be affected or mediated by confounding factors such as comorbidities (eg, diabetes, hypertension) and cigarette smoking, and a lack of adjustment for those factors might compromise the result.

In an effort to contribute to the existing literature and to complement the previous study on premenopausal women, ${ }^{11}$ we undertook this study to test the hypothesis that artherosclerosis is associated with osteoporosis. Specifically, we sought to define the relationship between cIMT and BMD in postmenopausal women and men aged 50 years and older of Vietnamese background.

\section{MATERIALS AND METHODS}

\section{Study design}

The present study was part of the Vietnam Osteoporosis Study (VOS), in which the protocol and design have been described elsewhere. ${ }^{12}$ Briefly, VOS is designed as a population-based longitudinal study, with the setting being Ho Chi Minh City (formerly Saigon), a major city in Vietnam. The overall goal of VOS is to define the relationship between osteoporosis and other non-communicable diseases. We used two approaches to recruit participants. In the first approach, we contacted community organisations to solicit a list of members, and from the list we ran a computer program to randomly select individuals who met the age and gender criteria. A letter was then sent to the selected individuals to invite them and their family members to participate in the study. In the second approach, we recruited participants via television, the internet and flyers in universities. The study has involved 4157 men and women aged from 20 to 90 years. In this study, we limited to those aged 50 years and older. The study was conducted according to the ethical principles of the Declaration of Helsinki, and written informed consent was obtained from each participant.

\section{Data collection and measurements}

Lifestyle factors and clinical history were ascertained via a structured questionnaire. Past and current smoking habits were assessed in terms of pack-years for each individual. Anthropometric parameters including body weight and standing height were obtained by measurements using an electronic portable, wall-mounted stadiometer (Seca Model 769; Seca, California, USA). The measurement of height was determined to the nearest $0.1 \mathrm{~cm}$ without shoes, and body weight was determined to the nearest $0.1 \mathrm{~kg}$ without shoes or heavy clothing. Body mass index (BMI) was defined as the ratio of weight $(\mathrm{kg})$ over the square of height $\left(\mathrm{m}^{2}\right)$. The individual's blood pressure was obtained as mean of two measurements (taken $10 \mathrm{~min}$ apart) in seated position. The individuals were classified as having hypertension if their average systolic blood pressure was $\geq 140 \mathrm{~mm} \mathrm{Hg}$ or diastolic blood pressure was $\geq 90 \mathrm{~mm} \mathrm{Hg}$.

Areal BMD was measured at lumbar spine (L1-L4), femoral neck and whole body using a dual-energy X-ray absorptiometry (DXA, Hologic Horizon). Calibration of densitometer was carried out using a standard phantom before each measurement. The measurement was done by a qualified radiology technologist. Based on 20 individuals, the coefficient of variation in femoral neck and lumbar spine BMD determined at our laboratory was between $1.5 \%$ and $2 \%$. We excluded spinal BMD data from 13 individuals with severe vertebral collapse, scoliosis degenerative changes and/or artefacts where accurate BMD could not be estimated.

Participants were categorised into the osteoporosis, osteopaenia and normal groups based on the WHO's recommended T-scores. In this study, the T-scores were calculated based on the femoral neck BMD reference range that has been published previously. ${ }^{13}$

\section{Carotid intima-media thickness}

cIMT was measured using Philips Ultrasound (model HD7XE) with a $7 \mathrm{MHz}$ linear transducer. The measurement was done at three segments of both the left and the right sides: a segment of $1 \mathrm{~cm}$ at the common carotid artery (CCA) $1 \mathrm{~cm}$ proximal to the flow divider, a segment of $1 \mathrm{~cm}$ at carotid bulb $(\mathrm{CB})$ proximal to the flow divider and a segment of $1 \mathrm{~cm}$ at internal carotid artery (ICA) proximal to the flow divider. cIMT is defined as the mean distance between two parallel thick echoes of the doubleline pattern of the far artery wall. The average of left and right $\mathrm{CCA}, \mathrm{CB}$ and ICA values (in $\mathrm{mm}$ ) for each subject was used for analysis. All measurements were performed by two qualified ultrasonographers in Tam Duc Heart hospital. The ultrasonographers were blinded to the clinical information. All images were stored as DICOM format and analysed using Philips' QLAB 11.0 software.

In addition, atherosclerotic carotid plaques were determined at six different locations: CCA, CB and ICA on both the left and right sides. Plaque was defined as a focal thickening that encroaches into the lumen by $0.5 \mathrm{~mm}$ or by $50 \%$ of the surrounding IMT or where IMT is $>1.5 \mathrm{~mm}$. In this study, we subjectively define plaques as present or absent for analysis.

\section{Biochemical analysis}

Blood sample was taken from each participant in the morning after an overnight fast for about 8 hours. The serum was immediately frozen to $-20^{\circ} \mathrm{C}$ prior to analysis which was done within 24 hours after collection. Serum levels of glycated haemoglobin (HbA1c) were measured using the high pressure liquid chromatography analysers ADAMS A1c HA-8160 (Arkray, Japan) with the intra-assay 
and interassay coefficient of variance of $<1 \%$. Glucose levels were measured by hexokinase test (Advia 1800 Autoanalyzer, Bayer Diagnostics, Germany). Total cholesterol and low-density lipoprotein cholesterol were determined by enzymatic calorimetric method (Advia 1800 Autoanalyzer, Bayer Diagnostics). The intra-assay coefficient of variation was $0.6 \%$. All samples were analysed at the MEDIC's Department of Biochemistry and Paraclinical Services (Ho Chi Minh City, Vietnam).

\section{Data analysis}

We conducted both descriptive and inferential analyses to address the main aims of the study. In the descriptive analysis, we computed mean and SD for each continuous variable stratified by sex. The individuals were then stratified into normal, osteopaenia and osteoporosis subgroups based on the femoral neck BMD measurement. ${ }^{13}$ The differences between these subgroups by BMD and sex were tested by the analysis of variance (for three-group comparison) or t-test (for two-group comparison) for normally distributed data or Pearson's $\chi^{2}$ test for categorical data.

We used the multivariable linear regression analysis to assess the relationship between cIMT and BMD. In this analysis, cIMT was considered the primary outcome, BMD was treated as a predictor variable and age, sex, clinical factors were covariates. The clinical risk factors included the presence/absence of diabetes, hypertension and cigarette smoking which were known to affect cIMT. Because the distribution of cIMT variables was not normally distributed, we transformed the variables into natural logarithmic scale before analysis. The strength of association between each predictor or covariate and cIMT was estimated by the regression coefficient and $\mathrm{SE}$. We further analysed the relative contribution of individual predictors to the variance in cIMT by the relative importance regression analysis method. ${ }^{14}$ For the association between the presence of plaque and BMD, the multivariable logistic regression model was used. All statistical analysis was performed using $\mathrm{R}$ statistical environment. ${ }^{15}$

\section{Patient and public involvement}

No patients and/or public were involved in the research design, and no patients and/or public were directly involved in the analysis of data.

\section{RESULTS}

This study involved 559 men and 901 women, whose baseline characteristics stratified by sex, are shown in table 1 . All individuals aged 50 years and older, with average age being $~ 59$ years. There was no statistically significant $(\mathrm{p}=0.92)$ difference in BMI between men and women. There were more men $(32 \%)$ than women $(25 \%)$ with hypertension. Using the criteria of HbAlc $\geq 6.5 \%$, the prevalence of diabetes in men and women was $14 \%$. About $40 \%$ of men and $0.8 \%$ of women were self-reported current smokers. Moreover, cIMT was significantly higher in men (mean $0.76 \mathrm{~mm}$ ) than women $(0.67 \mathrm{~mm})$.
Table 1 Baseline data on demographic and clinical characteristics of 901 women and 559 men aged 50 years and older, stratified by sex

\begin{tabular}{|c|c|c|c|}
\hline Parameter & Men & Women & P value \\
\hline $\mathrm{N}$ & 559 & 901 & \\
\hline Age (years) & $59.1(7.1)$ & $59.5(7.4)$ & 0.40 \\
\hline BMI $\left(k g / m^{2}\right)$ & $23.4(3.1)$ & $23.5(3.2)$ & 0.92 \\
\hline Hypertension (yes) & 179 (32.0\%) & $221(24.6 \%)$ & 0.02 \\
\hline $\begin{array}{l}\text { Diabetes mellitus } \\
\text { (yes) }\end{array}$ & $128(14.3 \%)$ & $80(14.3 \%)$ & 0.99 \\
\hline $\begin{array}{l}\text { Current smoking } \\
\text { (yes) }\end{array}$ & $224(40.1 \%)$ & $6(0.8 \%)$ & $<0.001$ \\
\hline $\begin{array}{l}\text { Total cholesterol } \\
(\mathrm{mmol} / \mathrm{L})\end{array}$ & $5.17(1.04)$ & $5.51(1.16)$ & $<0.001$ \\
\hline $\begin{array}{l}\text { LDL cholesterol } \\
(\mathrm{mmol} / \mathrm{L})\end{array}$ & $3.31(1.0)$ & $3.61(1.1)$ & $<0.001$ \\
\hline clMT (mm) & $0.76(0.24)$ & $0.67(0.16)$ & $<0.001$ \\
\hline Plaque (yes) & 309 (55.7\%) & 375 (42.1\%) & $<0.001$ \\
\hline
\end{tabular}

Values are shown in mean and SD (in bracket). $P$ values were derived from the t-test analysis. For categorical variables, data shown are number of individuals and percentage (in bracket) and $p$ values were derived from the Pearson's $\chi^{2}$ test.

Bold values signify statistical significance.

cIMT, carotid intima-media thickness; LDL, low-density lipoprotein.

Men also had a significantly higher prevalence of atherosclerotic plaques than women ( $56 \%$ vs $42 \%$; $\mathrm{p}<0.001)$.

$\mathrm{BMD}$ was grouped into three groups based on T-score classification: normal, osteopaenia and osteoporosis. The prevalence of osteoporosis in men and women was $16.6 \%$ $(\mathrm{n}=149)$ and $6.6 \%(\mathrm{n}=37)$, respectively. In women, the prevalence of atherosclerotic plaques was significantly higher $(\mathrm{p}<0.001)$ in the osteoporotic group $(56 \%, \mathrm{n}=83 / 149)$, almost twofold higher than that in the normal group $(33 \%$, $n=80 / 248$ ). In men, although the prevalence of atherosclerotic plaques was also higher in the osteoporotic group $(65 \%, \mathrm{n}=24 / 37)$ compared with that in the normal BMD group $(56 \%, n=139 / 249)$, the difference was not statistically significance $(\mathrm{p}=0.42)$ (data not shown).

There was a significant relationship between cIMT and age in both men and women (figure 1). This relationship is characterised by an almost linear increase in cIMT with advancing age. In either sex, each year increase in age was estimated to associate with a $0.01 \mathrm{~mm}$ (SE 0.001) thicker in cIMT. The correlation coefficient between cIMT and age was 0.38 for men and 0.34 for women. At each age, men had higher cIMT than women, and the sex-related difference appeared to increase with advancing age.

The association between cIMT and BMD was assessed by the multiple linear regression analysis (table 2). In men, higher cIMT was statistically significantly associated $(\mathrm{p}=0.015)$ with femoral neck BMD, after adjusting for age, cigarette smoking and type 2 diabetes. However, the proportion of variance in cIMT explained by femoral neck BMD was $0.5 \%$. Collectively, these factors-femoral 


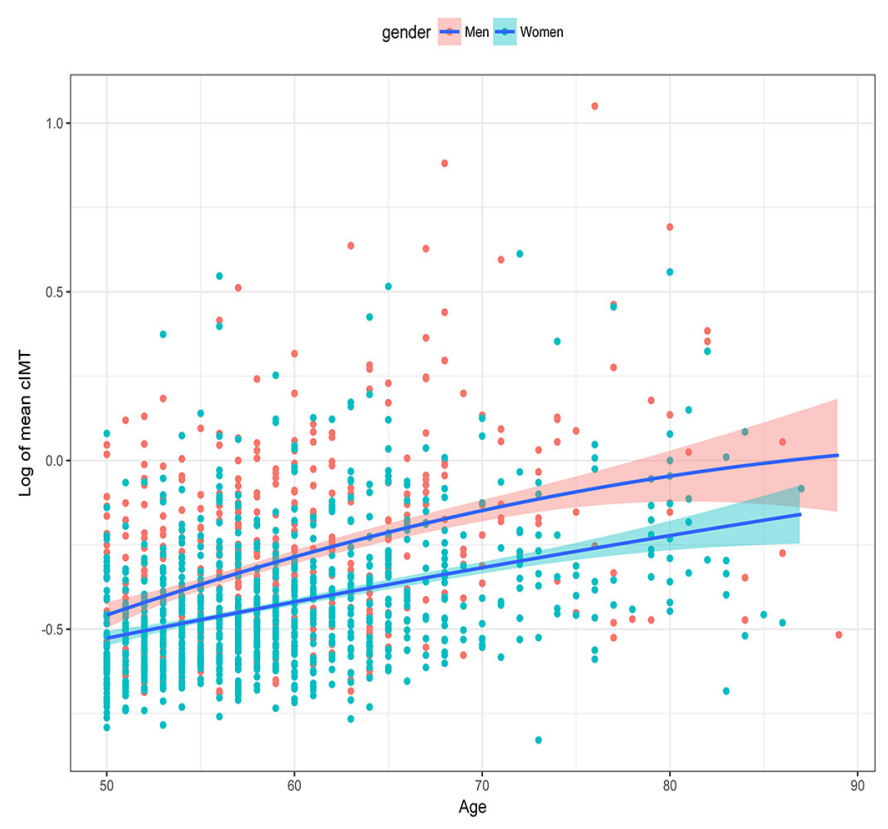

Figure 1 Association between carotid intima-media thickness (cIMT) with age for men (pink colour; $n=559$ ) and women (blue colour; $\mathrm{n}=901$ ).

neck BMD, age, cigarette smoking, type 2 diabetes, and hypertension-explained $19 \%$ of total variance in cIMT.

In women, there was no statistically significant association ( $\mathrm{p}=0.369)$ between cIMT and femoral neck BMD, after adjusting for advancing, type 2 diabetes and hypertension. The four factors collectively accounted for $\sim 17 \%$ of variance in cIMT, with almost $12 \%$ being due to advancing age.

We repeated the analysis for individual measures of IMT (eg, common carotid artery IMT, internal carotid artery IMT and carotid bulb artery IMT) stratified by sex (see online supplementary table 1). We also found that femoral neck BMD was statistically significantly $(\mathrm{p}<0.05)$

Table 2 Association between cIMT and femoral neck BMD stratified by gender

\begin{tabular}{|c|c|c|c|}
\hline Gender/Predictor & $\begin{array}{l}\text { Regression } \\
\text { coefficient (SE) }\end{array}$ & P value & $\mathbf{R}^{2}$ \\
\hline \multicolumn{4}{|l|}{ Men } \\
\hline Age (+5years) & $0.015(0.001)$ & $<0.001$ & 0.161 \\
\hline $\begin{array}{l}\text { Femoral neck BMD (g/ } \\
\left.\mathrm{cm}^{2}\right)\end{array}$ & $0.021(0.008)$ & 0.015 & 0.005 \\
\hline Diabetes (yes) & $0.074(0.028)$ & 0.009 & 0.012 \\
\hline Hypertension (yes) & $0.039(0.022)$ & 0.071 & 0.006 \\
\hline Smoking (yes) & $0.056(0.020)$ & 0.006 & 0.007 \\
\hline \multicolumn{4}{|l|}{ Women } \\
\hline Age (+5years) & $0.009(0.001)$ & $<0.001$ & 0.115 \\
\hline $\begin{array}{l}\text { Femoral neck BMD ( } \mathrm{g} / \\
\left.\mathrm{cm}^{2}\right)\end{array}$ & $0.053(0.059)$ & 0.369 & 0.008 \\
\hline Diabetes (yes) & $0.094(0.019)$ & $<0.001$ & 0.032 \\
\hline Hypertension (yes) & $0.051(0.015)$ & $<0.001$ & 0.017 \\
\hline
\end{tabular}

$\mathrm{BMD}$, bone mineral density; cIMT, carotid intima-media thickness. associated with common carotid artery IMT and internal carotid artery IMT in men, but not in women.

In a further analysis, we excluded 110 individuals with self-reported cardiovascular disease, and the results remained largely unchanged. When we limited the analysis to those aged 70 years and older, we found no statistically significant association between cIMT and femoral neck BMD ( $>0.3)$ or lumbar spine BMD ( $>0.5)$ (data not shown).

We further analysed the association between atherosclerotic plaque and BMD (figure 2). Although men and women with atherosclerotic plaque tended to have a slightly higher BMD than those without atherosclerotic plaque, the difference did not reach a statistical significance. The logistic regression analysis with atherosclerotic plaque being the outcome also confirmed the descriptive result shown in figure 2 . There was no statistically significant association between femoral neck BMD and the odds of having atherosclerotic plaque in either men or women (table 3).

\section{DISCUSSION}

Several lines of biologic evidence suggest that osteoporosis and atherosclerosis are linked through shared risk factors and common paraphysiological mechanisms. However, empirical epidemiological evidence for the linkage has been contentious. In this study of individuals aged $50+$ years, there was a statistically significant positive association between cIMT and BMD in men, but not in women. We conclude that these data do not support the association between subclinical atherosclerosis and osteoporosis. We reached this conclusion because (i) the statistical signal was weak $(\mathrm{p}=0.015)$ and was found only in a subgroup analysis; (ii) the magnitude of association between cIMT and BMD was very low and (iii) the inconsistent association observed in men and women.

Our null-association finding is unlikely due to the statistical power of the study. Our sample size was adequate to detect a modest correlation coefficient as low as 0.1. We also used a state-of-the-art ultrasonography and densitometer for the measurement of cIMT and BMD, respectively. Our finding is, in fact, consistent with previous studies in Asian populations which showed no statistically significant relationship between cIMT and BMD. ${ }^{9}$ Moreover, in a secondary analysis, we found no statistically significant association between the presence of carotid plaque and BMD, and this finding is also in line with previous studies. ${ }^{16}$

From a biologic point of view, the relationship between cIMT and BMD is expected to be inverse as observed in some studies, ${ }^{6}{ }^{17-20}$ but we found a positive association between cIMT and BMD, and this direction of relation is also agreeable with a previous study in premenopausal women of Caucasian background. ${ }^{11}$ A recent study ${ }^{21}$ showed that cIMT was associated with an increased risk of hip fracture despite being associated with higher BMD. Thus, it seems that the positive association between IMT and BMD does not translate into a lower fracture risk in those with high IMT, suggesting that the relationship between atherosclerosis and osteoporosis is unlikely to be mediated by BMD. 

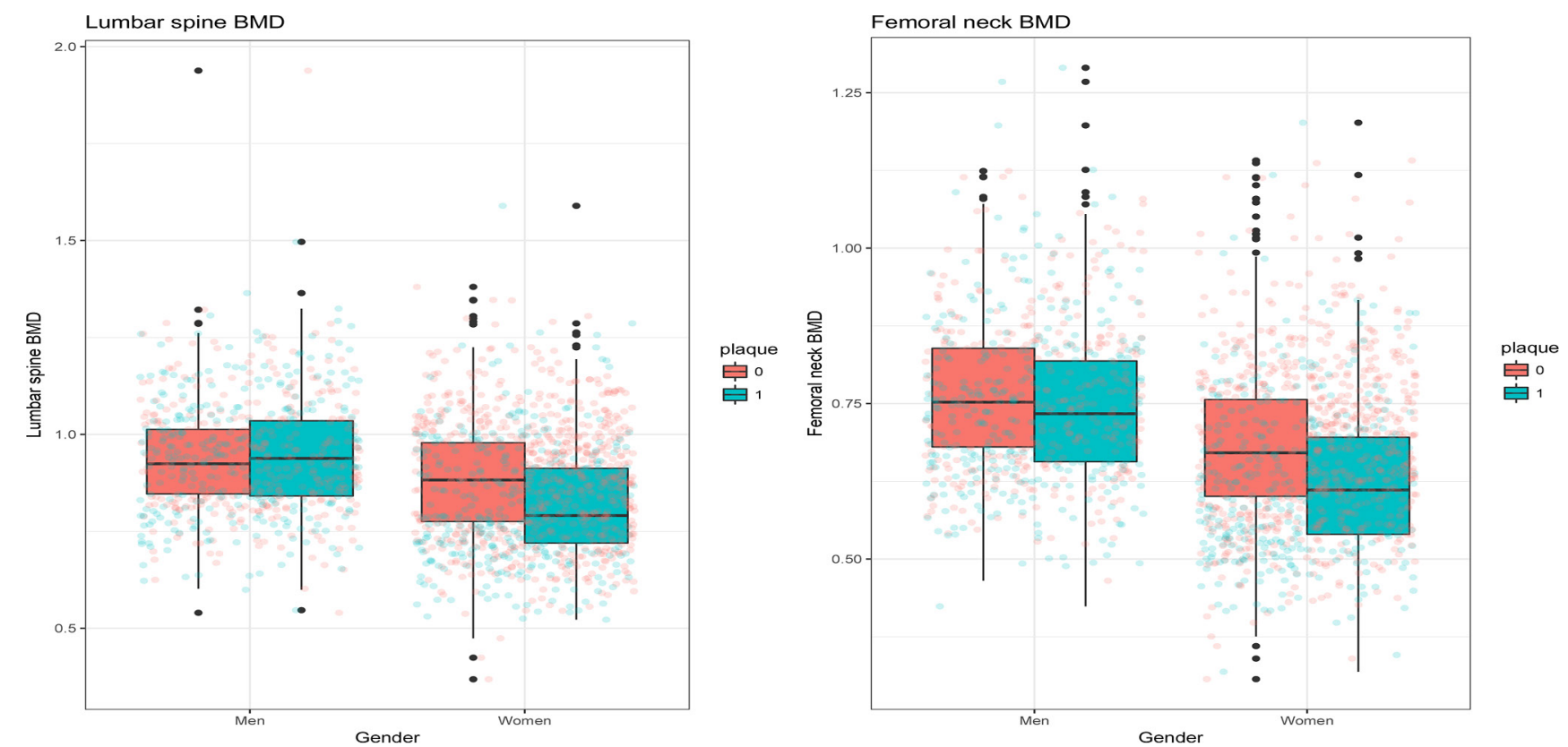

Figure 2 Association between presence of plaque and lumbar spine and femoral neck bone mineral density (BMD) stratified by gender.

Age appeared to be an important confounder in our study. In univariate analysis, we found that osteoporotic individuals had greater cIMT, but when age was taken into account, the difference was not statistically significant (in women) or even in opposite direction (in men). As expected, we found a significant age-related increase in cIMT in both sexes. However, it is interesting to observe that for a given age, cIMT in men was greater than women. The sex-related difference might be partly due to cigarette smoking in men, which in our study, was associated with a greater cIMT.

We note that men or women with type 2 diabetes on average had a greater cIMT than those without diabetes, and this finding is also consistent with previous studies. ${ }^{22} 23$ Moreover, as expected, individuals with hypertension also had greater cIMT, which confirms

Table 3 Association between presence of plaque and femoral bone mineral density (BMD) stratified by gender

\begin{tabular}{llr}
\hline Gender/Predictor & ORs $(95 \%$ Cl) & P value \\
\hline Men & & \\
\hline Age (+5years) & $1.11(1.08$ to 1.15$)$ & $<0.001$ \\
\hline Femoral neck BMD $\left(\mathrm{g} / \mathrm{cm}^{2}\right)$ & $1.14(0.93$ to 1.38$)$ & 0.198 \\
\hline Diabetes (yes) & $2.11(1.22$ to 3.65$)$ & 0.008 \\
\hline Hypertension (yes) & $1.08(0.73$ to 1.60$)$ & 0.698 \\
\hline Smoking (yes) & $1.65(1.13$ to 2.41$)$ & 0.009 \\
Women & $1.08(1.06$ to 1.11$)$ & $<0.001$ \\
Age (+5years) & $0.93(0.78$ to 1.10$)$ & 0.394 \\
\hline Femoral neck BMD $\left(\mathrm{g} / \mathrm{cm}^{2}\right)$ & $1.69(1.13$ to 2.54$)$ & 0.011 \\
\hline Diabetes (yes) & $1.12(0.8001 .56)$ & 0.507 \\
\hline Hypertension (yes) &
\end{tabular}

previous observations. ${ }^{24}$ We did not observe a relationship between cIMT and BMI. However, even after adjusting for these comorbidities and cigarette smoking, there was still no significant association between cIMT and BMD. The non-significant relationship was unchanged when we removed data of individuals with cardiovascular disease.

In the elderly, cIMT is a clinically relevant parameter, because it is associated with several risk factors for cardiovascular disease, including blood pressure, diabetes and smoking. ${ }^{25}$ Can BMD be used as an indicator of subclinical atherosclerosis in old people? Based on the present and previous findings, we consider that BMD does not adequately reflect atherosclerotic status either in the cIMT measurement or the presence/absence of atherosclerotic plaques. Even in previous studies where a statistically significant association between cIMT and BMD was observed, the magnitude of association was very modest, suggesting that the discriminatory value of BMD for atherosclerosis is not clinically useful.

Our findings should be considered within context of strengths and weaknesses. This study was based on a large and well-characterised cohort which was randomly recruited from the general population. While most previous studies showed association between cIMT and BMD in premenopausal women, this study provided complementary postmenopausal data and men aged 50 years and older. Although we have carefully adjusted for potential covariates, there may be unobserved confounders that have not been taken into account. This study did not consider other measures of atherosclerosis such as pulse wave velocity, aortic calcification index and endothelial function, which have previously been found to be associated with BMD. ${ }^{26-28}$ It should be noted that this is a cross-sectional study, and causal inference 
cannot be made for the association between carotid atherosclerosis and osteoporosis.

In conclusion, in this study of postmenopausal women and men aged 50 years and older, we found a statistically significant, but no clinically significant, association between cIMT and BMD in men, but not in women. This finding suggests that osteoporosis has little impact on atherosclerosis.

\section{Author affiliations}

${ }^{1}$ Bone and Muscle Research Group, Ton Duc Thang University, Ho Chi Minh City, Vietnam

${ }^{2}$ Faculty of Applied Sciences, Ton Duc Thang University, Ho Chi Minh City, Vietnam

${ }^{3}$ Tam Duc Heart Hospital, Ho Chi Minh City, Vietnam

${ }^{4}$ Department of Internal Medicine, Pham Ngoc Thach University of Medicine, Ho Chi Minh City, Vietnam

${ }^{5}$ Bone Biology Division, Garvan Institute of Medical Research, Sydney, New South Wales, Australia

${ }^{6}$ School of Biomedical Engineering, University of Technology, Sydney, New South Wales, Australia

Acknowledgements The authors would like to thank Ms Tran Thi Ngoc Trang and Fr Pham Ba Lam for coordinating the recruitment of participants. The authors would also like to thank doctors and medical students of the Pham Ngoc Thach University of Medicine for the data collection and clinical measurements.

Contributors TVN and LTH-P designed the study. LTH-P, LTN, VNP and PMNC collected the data. TVN, LTH-P and LTN were responsible for data analysis. LTN and PMNC prepared the first draft of manuscript. TVN and LTH-P revised manuscript. All authors approved the final version of manuscript.

Funding The Vietnam Osteoporosis Study was partly funded by Foundation for Science and Technology Development of Ton Duc Thang University (FOSTECT, http://fostect.tdt.edu.vn), grant number FOSTECT.2014.BR.09, and a grant from the Department of Science and Technology of Ho Chi Minh City.

Disclaimer The funders had no role in study design, data collection and analysis, decision to publish or preparation of the manuscript.

Competing interests LTH-P reports grants by Foundation for Science and Technology Development of Ton Duc Thang University (FOSTECT, http://fostect.tdt. edu.vn), grant number FOSTECT.2014.BR.09, and a grant from the Department of Science and Technology of $\mathrm{Ho}_{0}$ Chi Minh City during the conduct of the study.

\section{Patient consent for publication Not required.}

Ethics approval All procedures performed in this study were in accordance with the ethical standards of the Tam Duc Heart Hospital research committee.

Provenance and peer review Not commissioned; externally peer reviewed.

Data availability statement All data relevant to the study are included in the article or uploaded as supplementary information.

Open access This is an open access article distributed in accordance with the Creative Commons Attribution Non Commercial (CC BY-NC 4.0) license, which permits others to distribute, remix, adapt, build upon this work non-commercially, and license their derivative works on different terms, provided the original work is properly cited, appropriate credit is given, any changes made indicated, and the use is non-commercial. See: http://creativecommons.org/licenses/by-nc/4.0/.

\section{REFERENCES}

1. Browner WS, Pressman AR, Nevitt MC, et al. Association between low bone density and stroke in elderly women. The study of osteoporotic fractures. Stroke 1993;24:940-6.

2. Schoppet M, Sattler AM, Schaefer JR, et al. Increased osteoprotegerin serum levels in men with coronary artery disease. $J$ Clin Endocrinol Metab 2003;88:1024-8.

3. Anand DV, Lahiri A, Lim E, et al. The relationship between plasma osteoprotegerin levels and coronary artery calcification in uncomplicated type 2 diabetic subjects. J Am Coll Cardiol 2006;47:1850-7.

4. Abedin M, Omland T, Ueland T, et al. Relation of osteoprotegerin to coronary calcium and aortic plaque (from the Dallas heart study). Am J Cardiol 2007;99:513-8.
5. Kondo T, Endo I, Aihara K-I, et al. Serum carboxy-terminal telopeptide of type I collagen levels are associated with carotid atherosclerosis in patients with cardiovascular risk factors. Endocr $J$ 2016;63:397-404.

6. Hmamouchi I, Allali F, Khazzani H, et al. Low bone mineral density is related to atherosclerosis in postmenopausal Moroccan women. BMC Public Health 2009:9:388.

7. Frost ML, Grella R, Millasseau SC, et al. Relationship of calcification of atherosclerotic plaque and arterial stiffness to bone mineral density and osteoprotegerin in postmenopausal women referred for osteoporosis screening. Calcif Tissue Int 2008;83:112-20.

8. Kammerer CM, Dualan AA, Samollow PB, et al. Bone mineral density, carotid artery intimal medial thickness, and the vitamin $D$ receptor Bsml polymorphism in Mexican American women. Calcif Tissue Int 2004;75:292-8.

9. Yamada S, Inaba M, Goto H, et al. Significance of intima-media thickness in femoral artery in the determination of calcaneus osteosono index but not of lumbar spine bone mass in healthy Japanese people. Osteoporos Int 2005;16:64-70.

10. Liang D-K, Bai X-J, Wu B, et al. Associations between bone mineral density and subclinical atherosclerosis: a cross-sectional study of a Chinese population. J Clin Endocrinol Metab 2014;99:469-77.

11. Frysz M, Deere K, Lawlor DA, et al. Bone mineral density is positively related to carotid intima-media thickness: findings from a populationbased study in adolescents and premenopausal women. $J$ Bone Miner Res 2016;31:2139-48.

12. Ho-Pham LT, Nguyen TV. The Vietnam osteoporosis study: rationale and design. Osteoporos Sarcopenia 2017;3:90-7.

13. Ho-Pham LT, Nguyen UDT, Pham HN, et al. Reference ranges for bone mineral density and prevalence of osteoporosis in Vietnamese men and women. BMC Musculoskelet Disord 2011;12:182.

14. Grömping U. Estimators of relative importance in linear regression based on variance decomposition. Am Stat 2007;61:139-47.

15. The R Development Core Team. $R$ : a language and environment for statistical computing. Vienna, Austria: R Foundation for Statistical Computing, 2008. https://www.r-project.org/

16. Jørgensen L, Joakimsen O, Rosvold Berntsen GK, et al. Low bone mineral density is related to echogenic carotid artery plaques: a population-based study. Am J Epidemiol 2004;160:549-56.

17. Sumino H, Ichikawa S, Kasama S, et al. Relationship between carotid atherosclerosis and lumbar spine bone mineral density in postmenopausal women. Hypertens Res 2008;31:1191-7.

18. Värri M, Tuomainen T-P, Honkanen R, et al. Carotid intima-media thickness and calcification in relation to bone mineral density in postmenopausal women-the OSTPRE-BBA study. Maturitas 2014;78:304-9.

19. Mohammadi A, Shateri K, Behzadi F, et al. Relationship between intima-media thickness and bone mineral density in postmenopausal women: a cross-sectional study. Int J Clin Exp Med 2014;7:5535-40.

20. Shaffer JR, Kammerer CM, Rainwater DL, et al. Decreased bone mineral density is correlated with increased subclinical atherosclerosis in older, but not younger, Mexican American women and men: the San Antonio family osteoporosis study. Calcif Tissue Int 2007;81:430-41.

21. Barzilay JI, Buzkova P, Cauley JA, et al. The associations of subclinical atherosclerotic cardiovascular disease with hip fracture risk and bone mineral density in elderly adults. Osteoporos Int 2018;29:2219-30.

22. Matsagoura M, Andreadis E, Diamantopoulos EJ, et al. Carotid intima-media thickness in patients with type 2 diabetes: the significance of microalbuminuria and different risk factors for atherosclerosis. Diabetes Care 2003;26:2966.

23. Lorenz MW, Price JF, Robertson C, et al. Carotid intima-media thickness progression and risk of vascular events in people with diabetes: results from the PROG-IMT collaboration. Diabetes Care 2015;38:1921-9.

24. Zielinski T, Dzielinska Z, Januszewicz A, et al. Carotid intima-media thickness as a marker of cardiovascular risk in hypertensive patients with coronary artery disease. Am J Hypertens 2007;20:1058-64.

25. del Sol Al, Moons KG, Hollander M, et al. Is carotid intima-media thickness useful in cardiovascular disease risk assessment? the Rotterdam study. Stroke 2001;32:1532-8.

26. Sanada M, Taguchi A, Higashi Y, et al. Forearm endothelial function and bone mineral loss in postmenopausal women. Atherosclerosis 2004;176:387-92.

27. Hirose K-ichi, Tomiyama H, Okazaki R, et al. Increased pulse wave velocity associated with reduced calcaneal quantitative osteo-sono index: possible relationship between atherosclerosis and osteopenia. $J$ Clin Endocrinol Metab 2003;88:2573-8.

28. Farhat GN, Cauley JA. The link between osteoporosis and cardiovascular disease. Clin Cases Miner Bone Metab 2008;5:19-34. 